\title{
Reseña del libro History of Psychology in Latin America. A cultural approach
}

\author{
Fernando Ferrari ${ }^{1}{ }^{10}{ }^{2}$ \\ Universidad Nacional de Córdoba, Córdoba, Argentina.
}

\section{RESEÑA DEL LIBRO}

J. C. Ossa, G. Salas, \& H. Scholten (eds.) (2021). History of Psychology in Latin America. A cultural approach. . Springer. https://doi.org/https://doi.org/10.1007/978-3-03073682-8.

La editorial Springer, que tiene como objetivo estimular las producciones latinoamericanas en el mundo angloparlante, nos trae la publicación de un libro colectivo con ambiciosos y necesarios objetivos, editado por Hernán Scholten, Gonzalo Salas y Julio César Ossa.

Antes que nada, debo decir que emitir un juicio sobre esta producción no es sino un acto de arrojo, pues brindar una reseña ajustada a la calidad de sus producciones entraña una gran dificultad. En primera instancia, por el hecho de que cada capítulo comporta un análisis de diferentes microclimas culturales que, a su vez, dialogan con una historiografía específica con la que es difícil, sino imposible, estar a tono dada su amplitud.

El libro se divide en dos grandes apartados. La primera parte del libro, Notas generales sobre una historia de la psicología en Latino América, presenta trabajos en los que hay un esfuerzo de realizar estudios comparativos de diversos objetos vinculados a la historia de la psicología en diferentes países de Latinoamérica. En esto se distingue de la segunda instancia, Narrativas para una historia social, política y cultural de la historia de la psicología, en donde ya no hay trabajos comparativos sistemáticos sino un desarrollo de capítulos por países.

El primer capítulo, Historiografía de la psicología latino americana: Notas y desafíos, escrito por los editores del libro, funciona como una introducción general, pues se postulan allí los objetivos del emprendimiento editorial, al tiempo que se traza un

\footnotetext{
1 Correspondence about this article should be addressed Fernando Ferrari: ferrarijfernando@gmail.com

2 Conflicts of Interest: The authors declare that the research was conducted in the absence of any commercial or financial relationships that could be construed as a potential conflict of interest.
} 
estudio que explora la historiografía de la historia de la psicología Latinoamericana desde 1960 al presente. El objetivo es exponer las notas de una historia general de la psicología latinoamericana con especificidad en las narrativas que los países de la región comparten en común. Objetivo que no deja de lado la evidente diversidad que implica abordar un objeto tan complejo. Se alinea con una perspectiva crítica de la historia de la psicología que tiene en cuenta un descentramiento de las historias de la psicología en Estados Unidos y Europa, buscando una "historia local de la psicología" que contemple una historia policéntrica tal como proponen Brock (2014) y Danziger (2006). Se parte entonces de la idea de que "el modelo de centro y periferia es ya obsoleto y debería ser abandonado a partir de una aproximación policéntrica" es por ello que "más que pensar en una historia global o mundial de la historia de la psicología de la suma de historias locales, en su mayoría nacionales, buscamos enfatizar las articulaciones e interconexiones entre los desarrollos de diferentes centros" (p. 7). Este objetivo se satisfará en diferentes grados en cada uno de los capítulos que componen el libro, pues cada capítulo se plantea este objetivo desde reconstrucciones históricas diferentes entre sí. De hecho, podríamos decir que a partir de la segunda parte del libro este objetivo se cumple relativamente, puesto que, de hecho, se presentan contribuciones ordenadas desde un criterio nacional. En esta dirección, advertiremos que la complejidad de los temas abordados en los diferentes capítulos demanda una lectura con un estado del arte que se sumerja en realidades historiográficas muy diferentes. Por ello es que es muy difícil evaluar en todas sus consecuencias cada uno de los capítulos. De hecho, cada capítulo aplica a su modo lo que entiende por una historia cultural, crítica y policéntrica y cada capítulo procede iniciando su reconstrucción desde diferentes fuentes primarias, lo cual marca variaciones metodológicas y técnicas que modulan con cierta amplitud lo que podría reconocerse explícita o implícitamente, pues no en todos los capítulos se define abiertamente, como un estudio crítico de historia cultural, policéntrico y decolonial.

Los tres capítulos restantes de esta primera parte de libro abordan objetos muy disímiles entre sí, pero que tienen en común el esfuerzo por realizar una reconstrucción histórica comparativa. Este esfuerzo no es menor, pues gran parte de los trabajos que van a definir la segunda parte del libro brindan reconstrucciones de tipo mosaico, aportando problemas, fuentes y abordajes que se circunscriben a diferentes países latinoamericanos en compartimentos que no necesariamente se comunican o logran lanzarse a un abordaje comparativo. 
En el capítulo dos, Marina Massimi presenta Los Jesuitas en la historia de la psicología Latino Americana. Para ello realiza un análisis que cubre los inicios de la presencia de los jesuitas en territorio americano desde 1567, reconstruyendo las políticas de las reducciones y cómo se fueron implementando en territorio americano. Luego, al analizar la psicología filosófica de los jesuitas, delimita sus principales textos animados por la perspectiva antropológica del cristianismo mediante la doctrina aristotélico tomista. Aborda el proceso de construcción de conocimiento que se dio entre los nativos y los padres jesuitas que arriban desde el viejo continente. En la reconstrucción histórica se resalta la aplicación de categorías psicológicas, a partir del análisis de fuentes primarias en gran parte editadas (textos y misivas), a fenómenos extraños a la cultura occidental, tales como la antropofagia, entendiéndola como apetitos sensitivos orientados a objetos inapropiados o incluso movimientos de apetitos intelectuales. O también descripciones como bestialidad o acciones de poderes demoníacos que terminan en locura. Lamentablemente, en la traducción al idioma inglés no se conserva la terminología original, lo cual puede dificultar la posibilidad de comprender la aplicación de una red semántica a una serie de fenómenos extraños a la cultura occidental de origen europeo. A pesar de realizar un análisis de un objeto complejo en su extensión tanto temporal como espacial, el capítulo logra satisfacer uno de los objetivos planteados en el capítulo introductorio: explorar, a partir de misivas y escritos, el proceso de aplicación de una serie de categorías exportadas desde la cultura occidental a una serie de fenómenos que resultaban culturalmente extraños, todos ellos experimentados en el proceso de cristianización y colonización cultural que los jesuitas llevaron a cabo. El capítulo termina un tanto abruptamente sin realizar un balance general o una articulación entre las partes del capítulo, tampoco entre los capítulos del libro.

En el capítulo tres, Fernando Polanco, Josiane Sueli Beria, Martín Zapico y Rodrigo Lopez Miranda abordan Una Historia comparativa de la psicología durante las dictaduras en Sud américa (1964-1985). El capítulo está claramente orientado a un lector angloparlante, probablemente poco instruido acerca de la psicología en Sud América, indicándole la localización geográfica de la región y explicando el predominio del portugués y el español, dada su historia de colonización. Abordan el problema de la posición crítica en la reconstrucción histórica e introducen el cuestionamiento sobre el punto de vista del historiador, quizás por el hecho de que el objeto de estudio al que refieren su reconstrucción histórica implica una cercanía temporal con una más evidente politización de la lectura, tanto del historiador como de la disciplina de la psicología. Así 
se lee el rol marcado desde Martín Baró, en el que hay una posición política en la reescritura de la historia que busca definir una identidad y eventualmente una soberanía de los eventos por venir. Presenta una lectura crítica que busca una re-politización de la historia de la psicología, que debe regirse por un trabajo riguroso de archivo y una sofisticación del análisis hermenéutico. La reconstrucción tiende sus límites en un análisis de las dimensiones académicas de la psicología durante el periodo dictatorial de 1956 a 1976, en un ejercicio de comparación de los procesos observados en diferentes países, en particular Paraguay (1954), Bolivia (1964), Brasil (1964), Chile (1973), Uruguay (1973) y Argentina (1976). Luego de una apretada síntesis, que responde a cada uno de los países, en la que se delimita la agenda institucional de la disciplina psicológica y se presentan las características generales de las particularidades, se procede a dos necesariamente breves apartados que se ocupan de los refugiados y el exilio en psicología. En estos dos apartados, al implicar el desplazamiento de actores en el mapa sudamericano, se da con mayor naturalidad una articulación que no está tan presente en los apartados previos, en los que el análisis está preponderantemente desarrollado por cada país. En la lectura del capítulo se me presentaba la idea de que el contenido de cada apartado implicaba un desarrollo mucho más amplio y que el capítulo era una presentación de un programa de investigación, algo que hacia el final los mismos autores terminan por hacer explicito (p. 59). Este artículo es, de hecho, un planteo programático que no deja de ser introductorio para un proyecto que, evidentemente, demandará varios años de trabajos sistemáticos si se buscan satisfacer las premisas metodológicas que se postulan al inicio del capítulo. De hecho, este planteo se puede extender en muchos sentidos al proyecto editorial en su conjunto.

El cuarto capítulo, El desarrollo de la psicología en Latino américa: Contextos geopolíticos y procesos psicosociales, desarrollado por Walter Arias Gallegos, Ramón León y Mitchell Clark, busca analizar las circunstancias sociopolíticas que han dado forma a la psicología latinoamericana, dándole una particular identidad con respecto a la psicología que se ha desarrollado en otras partes del mundo"(p. 64). Su centro de atención son los contextos geopolíticos y los procesos psicosociales que han estimulado o impedido el desarrollo de la psicología. El texto se dedica a un análisis dividido por países y se ocupa de un complejo y extenso período, cuya delimitación merecería precisarse. Por lo general, el texto pivotea desde los inicios de la psicología a fines de siglo XIX desplazándose a grandes pasos hasta fechas contemporáneas. Este análisis comienza con un recorrido por la historia de la psicología en Argentina, con algunas 
imprecisiones históricas menores -por ejemplo, la Asociación Argentina de Psicoanálisis, no fue fundada en 1945, sino en 1942 (p.67). En el caso de Bolivia, también presenta un desplazamiento temporal muy amplio, que se inicia a partir de Georges Roume en 1919, y, muy rápidamente, se desplaza hacia las décadas de los 1970s y 1980s. Algo similar puede observarse en el caso del análisis de Brasil que inicia con los primeros asilos creados en Rio de Janeiro en 1854. También en el caso de Chile se puede advertir esta aproximación que toma grandes rasgos de la historia chilena de la psicología. De modo tal que el capítulo permite apreciar ciertas dificultades en torno a la aplicación de periodizaciones más o menos homogéneas y criterios narrativos uniformes a los casos estudiados. Además, las tesis que presentan los autores respecto de la relación entre el contexto político-económico y el desarrollo de la psicología como ciencia y profesión que mostraría a una psicología desconectada de los problemas psicosociales3- podría contrastarse con algunos casos particulares (la difusión de la psicotecnia durante el primer peronismo en Argentina) o incluso con el abordaje historiográfico que está orientado por una periodización centrada en la articulación entre gobierno y psicología (Rossi, Ibarra, Ferro: 2018; Rossi, 2000).

Por otra parte, llama la atención que se afirme que "La Psicología Latinoamericana, no ha visto los avances científicos que ocurrieron en los Estados Unidos o en varios países europeos dadas una serie de vicisitudes ideológicas, religiosas, sociales y políticas." (p.64). Esto contrasta con una referencia a Kurt Danziger, en la que justamente se valora el hecho de que su aproximación crítica a la psicología ha permitido cuestionar el dominio hegemónico del conocimiento científico moldeado por el pensamiento occidental. El solo hecho de pensar a la psicología latinoamericana como carente de los avances que ocurrieron en Estados Unidos u otros países europeos riñe con la cita a Danziger, que, por otra parte, también cuestiona la bipartición entre historia interna y externa. En este sentido, Danziger propone la categoría de interés intelectual, que define el punto de encuentro entre los intereses sociales y las estructuras cognitivas

\footnotetext{
3 "With few exceptions, such as during the Pinochet dictatorship in Chile, the political and economic context has had little or nothing to do with the development of psychology as a science and profession. While military governments and dictatorships were in power, psychology in Latin American countries continued with the processes of institutionalization and professionalization. This tendency provides an indication of how weak the democracies in Latin America are, but also demonstrates the disconnection of psychology as a discipline, from the psychosocial problems. On the other hand, this historical analysis also reveals that psychology has seen little consideration from the governments as a potential source for solutions to the socio-economic problems of the inhabitants." (p.83)
} 
y permitiría superar la separación tajante entre "factores sociales" y "contenidos intelectuales".

A partir de la segunda parte del volumen, Narrativas para una historia cultural, política y social de la psicología, los capítulos ya comienzan a sucederse con un modo de trabajo que no sostiene aproximaciones comparativas. Mas bien se procede por colaboraciones que sitúan a la historia de la psicología en diversos países de Latinoamérica: Argentina, Brasil, Colombia, Paraguay, Chile, Perú y México.y que, en todo caso, aspiran a que el lector anglosajón pueda disponer de una serie de recortes ilustrativos de la historia en cada uno de los países. Este abordaje se distancia en cierto sentido de los objetivos que se trazaban en el primer capítulo -esto es, brindar una serie de problematizaciones en torno a la historia latinoamericana que se vería mucho más enriquecida con abordajes comparativos. No obstante, esta serie de capítulos, sí logran acercar a un público angloparlante, muestras y recortes de una historia latinoamericana de la psicología que inducen a un ejercicio de comparación que bien podría ejecutarse en el virtual lector. Como anunciamos anteriormente, es claro que en cada uno de los capítulos se desarrolla una historia cultural de la psicología que, en todo caso, es definida en su diversidad. De modo que, en cada contribución circunscripta a un recorte geográfico, se ejecuta una reconstrucción que atiende a objetos de estudio diversos, definidos por fuentes primarias y problemas también diversos.

En el caso del capítulo cinco, Cultura, política y sociedad en la historia de la psicología en Argentina, escrito por Hugo Klappenbach y Catriel Fierro, se aborda este último punto partiendo de una apropiación crítica de la perspectiva de la historia cultural, postulando que es necesario definir: "qué podría ser comprendido por una historia cultural de la psicología en Argentina. Tal definición podría probablemente estar cercana al uso extendido realizado de la psicología por grandes partes de las poblaciones sociales y los diferentes espacios de nuestra vasta geografía. Pero con algunas excepciones de algunos estudios aislados, tal historia cultural todavía permanece sin ser escrita" (p. 95). Y, justamente, se plantea una serie de problemas para definir el abordaje metodológico de una historia cultural de la psicología. Basándose en una periodización ya bastante conocida, los autores revisitan los inicios de la psicología en Argentina, un período que ha sido muy estudiado, en el cual sus principales características han sido ya establecidas y estabilizadas hasta el período de los años 1960s. Sin embargo, se advierte una novedad en la incorporación de un apartado que aborda un período que abarca desde la década de 1980 hasta casi la actualidad (1984 a 2009), en donde el campo de la historia reciente 
presenta sus aportes en un campo todavía en constitución y discusión. En este último apartado la reconstrucción se centra específicamente en el aspecto institucional de la conformación de las políticas universitarias. Mientras la periodización integra estos períodos más recientes, los autores se centran mucho más en algunos aspectos característicos del sílabo en la formación en psicología actual y en los mecanismos de evaluación institucional por los que la carrera de psicología ha atravesado los últimos años.

En el capítulo seis, La psicología en Brasil: trayectoria de una ciencia y una profesión, escrita por Ana María Jacó-Vilela, se parte de la hipótesis de que la historia de la psicología en Brasil no presenta grandes diferencias con la historia de la psicología en otros países periféricos de América Latina, Asia, y África. Esto implica para la autora dos cosas: por un lado, que la emergencia de la ciencia tiene origen en procesos que Europa atravesó en el siglo XV, y que ocurre en países en los que eventualmente se hizo una fuerte inversión en la creación de universidades y condiciones necesarias para la investigación. Por otro lado, la psicología emerge específicamente como un resultado de profundos cambios en las grandes ciudades, el incremento sociodemográfico y los avances en comunicaciones y tecnología. El capítulo se propone una visión general de la emergencia y desarrollo de la psicología como ciencia y profesión en Brasil, partiendo de una serie de publicaciones ordenadas por investigaciones previas. En esta línea es que se aborda un período extenso iniciando en el ingreso de Brasil al siglo XIX, a partir de una primera recepción de las teorías psicológicas permeadas por las teorías darwinianas de la selección natural. Las teorías frenológicas de Gall y las de Galton sobre la herencia que marcaron a la ciencia brasilera en orden de justificar conductas de dominación en torno a los procesos de colonización, por un fuerte aval de la esclavitud como política económica que sostenía la relación entre Portugal y Brasil. El trabajo pasa luego a abordar la aplicación de los primeros test psicológicos en el temprano siglo XX, a partir de la creación del primer laboratorio de psicología experimental en 1906 por Manoel Bomfim. Hacia el final del capítulo, los últimos apartados despliegan apreciaciones del estado actual de la psicología en Brasil y se plantean una serie de preguntas que buscan articular éticamente la actualidad con el decurso histórico de la disciplina. Algo que decanta una breve conclusión.

En el capítulo siete, La psicología en Colombia: Una historia de violencia, escrito por Juan Aguilar, Julio césar Ossa, Claudia Burbano y Jean Nikola Cudina, se aborda la relación de la psicología colombiana con el contexto social cultural y político. El capítulo 
plantea, con una coloración ensayística, cuatro períodos en los cuales la psicología constantemente aparece entrelazada a una serie de eventos institucionales y políticos que surgen de la particular historia colombiana, que inician con la creación del Instituto de Psicología Aplicada, creado en 1947, hasta la actualidad. Los cuatro períodos se proponen implican: 1- El colonialismo intelectual de la psicología colombiana que enfrenta a una ola de violencia (1948-1960); 2- La configuración de una psicología que enfrenta una realidad del conflicto armado interno (1961-1980); 3- la apuesta por una dimensión política de la psicología colombiana (1981-1990) y 4- la evolución de la psicología de frente a una Colombia postconflicto (1991-2020). El texto quiere mostrar cómo la psicología tomó forma en el campo de la especialización a partir de demandas de diferentes sectores de la sociedad -algo que se distancia, en alguna medida, con algunas conclusiones vertidas en el capítulo cuatro. En todo caso, es interesante el ejercicio de diálogo que suscita la lectura de los diferentes capítulos del libro.

En el capítulo ocho, La aproximación psicológica al estudio del hombre peruano originario, escrito por Tomás Caycho-Rodríguez, Miguel Barboza-Palomino y José Ventura-León, sitúa su problema luego de hacer un breve recorrido a partir de la distinción entre historias clásicas e historia crítica y de ubicar la contribución en torno a la historia crítica: una revisión sobre la explicación y características de la conducta del hombre originario peruano en el período colonial y el temprano siglo XX. Un período extenso representado entre dos puntos, por un lado, las representaciones que se extraen de los textos del médico doctor y naturalista Hipólito Unanue (1755-1833) Observaciones sobre el clima de Lima y su influencia en los seres organizados, en especial el hombre. El mismo es contextualizado en el marco de la introducción de un movimiento iluminista caracterizado por el racionalismo de Descartes, las matemáticas y físicas de Newton y la filosofía de Leibniz, Locke el empirismo y el sensualismo de Condillac. Cambios que se traslucieron también en el campo de la psicología, donde cuestiones como la naturaleza del alma, la inmortalidad, relaciones entre el cuerpo, la voluntad, las sensaciones, la memoria y la imaginación se abordaron ampliamente. El contrapunto temporal es demarcado a partir de las ideas de Valdizán (1885-1929) quien es el punto de inicio de los estudios sobre la degeneración en el Indio Peruano. Valdizan estudió en Europa junto a Sante de Sanctis. Tras su formación aplicó y difundió el psicoanálisis y otras teorías psicológicas. Actuó como un experto forense aplicando test de inteligencia y se mostró interesado en estudios del niño mentalmente deficiente. Para ello, instaló un laboratorio de psicología experimental en 1919 en el Asilo Colonia Magdalena. Además de su 
alineamiento con el positivismo, Valdizán mostró interés en el trabajo psicopatológico que desarrolló Karl Jaspers. Si bien el estudio muestra las lecturas que ambos autores realizan sobre el Hombre Peruano, no hay muchas referencias a un trabajo conceptual que compare con los referentes originales a los fines de advertir lecturas particulares, o interpretaciones singulares de la aplicación de las grillas nocionales de referencia.

El capítulo nueve, Psicología, historia y cultura en Paraguay, escrito por José García, inicia con un cuestionamiento en torno a los criterios de periodización que rodean a la historia de la psicología, y las dificultades de realizar una historia local. En particular la del Paraguay, debe tomar en cuenta la compleja trama cultural que precede al arribo de la cultura española, que aloja una diversidad de diecisiete etnias que coexisten, cada una de ellas con sus diferentes historias y tradiciones. En un texto panorámico sobre la historia de la psicología en el Paraguay, inicia desde el período colonial hasta el establecimiento de la psicología como un campo disciplinario profesionalizado en la década de 1960. En el primer apartado aborda los períodos coloniales y los tiempos previos a la colonización. Explora el modo en que los españoles generaron una serie de políticas como la encomienda, como parte de una política de esclavitud o dependencia. En este marco las creencias de los indígenas, o aborígenes nativos guaraníes, se vieron drásticamente reinterpretados y distorsionados por la mentalidad de los conquistadores, quienes tuvieron por intención principal importar los modelos españoles de filosofía y teología con los valores del catolicismo, en particular las Jesuitas quienes implementaron varias misiones en el Paraguay. Esta contribución nos recuerda los temas tratados por Massimi en el capítulo dos que, sin embargo, no encuentra una articulación en este texto, lo cual podría ser de gran valor. Por el contrario, este capítulo, al igual que otras contribuciones, parece presentarse sin una comunicación con el resto del libro. La historia del Paraguay trazada en el capítulo también comparte algunos solapamientos con la historia argentina, por ejemplo, se mencionan algunas referencias a los conflictos que se dieron con las políticas anexionistas del gobierno de Buenos Aires por parte de Juan Manuel de Rosas o cuando se analizan los textos del Dr. José Gaspar Rodríguez de Francia (1776-1840), graduado en teología sagrada en la Universidad de Córdoba del Tucumán (Argentina). Estos solapamientos exploran algunos aspectos que pueden ser una interesante puerta para explorar eventuales vasos comunicantes en una aproximación comparativa que muestre la circulación del conocimiento en diferentes microclimas culturales. Algo que se toca en el trabajo, pero que finalmente cede ante la inercia propia de las fuentes que tiende a resolverse en una reconstrucción dentro del marco de lo nacional. 
El capítulo diez, Psicología y educación: Higiene Mental infantil en Chile (19201946), escrito por Gonzalo Salas, Hernán Scholten y Rodolfo Mardones, se centra en una reconstrucción de la historia de la psicología en Chile, que toma por recorte una reconstrucción de las infancias. Particularmente, se apunta a definir y caracterizar la manera en que la infancia fue intervenida a partir del arribo de la Higiene Mental en Chile, a inicios de la década de 1920, desde una perspectiva local que no abandona las conexiones internacionales de lo que se nominó como la higiene mental infantil o la higiene mental escolar, que apuntaba a intervenir y solucionar los problemas suscitados por los niños que transitaban los márgenes de la normalidad y la locura y que eran considerados "mal adaptados". Se busca estudiarla desde una perspectiva psicológica y educacional enfatizando aspectos culturales e institucionales. Si bien el trabajo está circunscripto a lo sucedido en Chile, hay momentos en los que la reconstrucción se permite articular el decurso de la historia de la higiene mental en Brasil y en Argentina.

Hacia el final, en el capítulo once, Una fuente diferente para el estudio de la psicología en México: La educación católica, trazado por Iván Rodríguez Preciado, se aborda el problema de la historia de la psicología en México a partir de fuentes ligadas a la educación católica, tomando una opción metodológica que aporta a los estudios previos que exploran las tempranas enseñanzas de la psicología en México a partir de la Escuela Nacional Preparatoria. De tal modo que se permite explorar la articulación de la psicología con la pedagogía y los discursos filosóficos del catolicismo mexicano, en particular estudiando las lógicas de introducción de ideas a partir de editoriales españolas o libros de psicología publicados a través de editoriales católicas mexicanas. Esta aproximación es muy interesante, en Argentina también hay una serie de sesgos que muchas veces dejan de lado el estudio del impacto de los textos, instituciones y políticas del campo religioso, que tuvieron un impacto diferencial dependiendo del microclima cultural en donde se despliega su influencia. De hecho, gran parte de los procesos de colonización en toda Latinoamérica estuvieron atravesados por las diferentes y complejas vertientes del catolicismo, algo que adquiere notoriedad en algunas de las producciones de este libro, pero que no es usualmente estudiado de manera sistemática por la historiografía de la psicología latinoamericana. Gran parte de las veces, las reconstrucciones históricas tienen a centrarse en la recepción de las ideas del positivismo y la ciencia dejando de lado las complejidades y variantes del diálogo con psicologías de corte filosófico-religiosas. Aseveración que también debe tomarse con cautela puesto que 
habría que situarse en un análisis mucho más sustentado de la compleja y disímil historiografía de la psicología en Latinoamérica.

Pues bien, a modo de valoración general de la experiencia de lectura del libro, podemos aseverar que estamos ante un ejercicio valorable que pone sobre el telón de la discusión a problemas de difícil y necesaria discusión: ¿Qué queremos decir cuando decimos historia cultural de la psicología? ¿Qué premisas asumimos cuando nos comprometemos con una historia crítica de la psicología? ¿Cómo pensamos el problema del cuestionamiento a una historia centralista en oposición a una historia policéntrica de la psicología? ¿Qué problemas metodológicos, técnicos y teóricos implican todas estas interrogantes? ¿Qué es y cómo se aborda una historia cultural, crítica, policéntrica, decolonial de la psicología latinoamericana? ¿Por qué sesgo abordar tremendo problema? Está claro que este libro lanza muchos desafíos por venir. Sobre todo, abre el campo todavía no constituido de discusiones en torno a los estudios comparados de la historia de la psicología latinoamericana. Como efecto inmediato luego de la lectura del libro, surgieron una serie de cuestiones en torno a cómo abordar colaborativamente este tipo de estudios. El esfuerzo por comenzar a estabilizar un campo de problemas que esté favorecido por proyectos subvencionados de equipos de investigación internacionales que se mantengan en el tiempo. La conformación de objetos de estudio más homogéneos, particularmente estructurados por superficies documentales compartidas, que permitan un ejercicio de comparación de las diferencias de los microclimas culturales en donde se desplazan los conceptos y prácticas de las psicologías. El recorte temporal y la discusión de las periodizaciones, también animadas por el problema de una historia o unas historias de la psicología latinoamericana. Pues es todo un campo de cuestiones que este libro tiene la virtud de exponer al tiempo que anima al diálogo y al debate crítico. En este sentido, si bien, como habíamos mencionado, en el primer capítulo se busca explícitamente adherir a una historia crítica que busque el descentramiento desde una perspectiva policéntrica, llama la atención que el libro haya sido publicado exclusivamente en inglés. Es claro que la estrategia de la editorial es que las investigaciones latinoamericanas puedan llamar la atención del lector anglosajón, sin embargo, es de lamentar que su edición exclusivamente en idioma inglés, y el costo del volumen, dificulten el acceso a un amplio sector del público iberoamericano.

Por cuanto el texto atraviesa estas paradojas, proponiendo un proyecto ambicioso, complejo y necesario, puesto que su lectura no deja de evidenciar la necesidad de emprendimientos colaborativos transnacionales que se permitan el difícil análisis 
comparativo, crítico y decolonial en historia de la psicología latinoamericana. Algo que también nos induce al esfuerzo de intentar superar las inercias de formación de una historia localista que vaya más allá de los estudios que delimitan sus horizontes narrativos con los límites de lo nacional. Es por ello que las limitaciones que este libro pudiese presentar no empañan el efecto que produce su lectura de advertir la necesidad de la gestación del problema de la historia de la psicología en Latinoamérica como un objeto de estudio todavía en ciernes. En este sentido, este libro es claramente un aporte innovador, sugerente, que marca una agenda de investigaciones necesaria y problemática.

\section{Referencias}

Rossi, L., Ibarra, F., \& Ferro, C. (2008). Cambios políticos y psicología en Argentina. Revista de Historia de la Psicología en Argentina, 1, 52-63.

Rossi, L. (2000). 4. Genealogía de tradiciones conceptuales en psicología, su valoración en el marco político, social e institucional y su impacto en la conformación de la identidad profesional. En Psicología: su inscripción universitaria como profesión. Eudeba.

Brock, A. C. (2014). What is a polycentric history of psychology? Estudos e Pesquisas em Psicologia, 14(2), 646-659.

Danziger, K. (2006). Universalism and indigenization in the history of modern psychology. In A. C. Brock (Ed.), Internationalizing the history of psychology (pp. 208-225). New York University Press. 